\title{
ER stress-linked autophagy stabilizes apoptosis effector PERP and triggers its co-localization with SERCA2b at ER-plasma membrane junctions
}

\author{
Samantha J. McDonnell', David G. Spiller ${ }^{2}$, Michael R. H. White ${ }^{3}$, lan A. Prior (1D ${ }^{4}$ and Luminita Paraoan (D)
}

\begin{abstract}
Specific molecular interactions that underpin the switch between ER stress-triggered autophagy-mediated cellular repair and cellular death by apoptosis are not characterized. This study reports the unexpected interaction elicited by ER stress between the plasma membrane (PM)-localized apoptosis effector PERP and the ER $\mathrm{Ca}^{2+}$ pump SERCA2b. We show that the p53 effector PERP, which specifically induces apoptosis when expressed above a threshold level, has a heterogeneous distribution across the PM of un-stressed cells and is actively turned over by the lysosome. PERP is upregulated following sustained starvation-induced autophagy, which precedes the onset of apoptosis indicating that PERP protein levels are controlled by a lysosomal pathway that is sensitive to cellular physiological state. Furthermore, ER stress stabilizes PERP at the PM and induces its increasing co-localization with SERCA2b at ER-PM junctions. The findings highlight a novel crosstalk between pro-survival autophagy and pro-death apoptosis pathways and identify, for the first time, accumulation of an apoptosis effector to ER-PM junctions in response to ER stress.
\end{abstract}

\section{Introduction}

The p53 apoptosis effector related to PMP-22 (PERP) is a tetraspan plasma membrane (PM) protein that is involved in cell-cell adhesion and the regulation of apoptosis in many cell types ${ }^{1-3}$. PERP transcription is tightly controlled by both p53 and p63 and is specifically induced during p53-mediated apoptosis ${ }^{1,4}$. Expression of PERP above a threshold level correlates with the activation and stabilization of its regulator p53 and the cleavage of both caspase 8 and Bid; thus PERP positively influences its own expression and mediates apoptosis engaging both the extrinsic and mitochondrial pathways ${ }^{5,6}$. PERP is therefore likely to be a key molecule that drives damaged cells towards apoptosis ${ }^{7}$.

\footnotetext{
Correspondence: Luminita Paraoan (Iparaoan@liverpool.ac.uk)

${ }^{1}$ Department of Eye and Vision Science, Institute of Ageing and Chronic

Disease, University of Liverpool, Liverpool L7 8TX, UK

${ }^{2}$ Systems Microscopy Centre, Faculty of Biology, Medicine and Health,

University of Manchester, Manchester M13 9PT, UK

Full list of author information is available at the end of the article.

Edited by I. Lavrik
}

Protein synthesis and modification in the endoplasmic reticulum (ER) are highly dependent on a high luminal $\mathrm{Ca}^{2+}$ concentration. In addition, spatiotemporal $\mathrm{Ca}^{2+}$ signals, generated via release from the ER stores and influx from the extracellular space, regulate many cellular processes including autophagy and cell death ${ }^{8}$. Sarco/ endoplasmic reticulum ATPase (SERCA) pumps regulate $\mathrm{Ca}^{2+}$ influx into the ER and are vital for the maintenance of intracellular $\mathrm{Ca}^{2+}$ homoeostasis. The three SERCA genes (ATP2A1-3) undergo alternative splicing to generate a family of isoforms with tissue-specific expression $^{9,10}$. SERCA2 is expressed ubiquitously and is recruited to points of contact between the ER and PM involved in the fundamental store operated calcium entry (SOCE) influx mechanism, where it ensures efficient ER $\mathrm{Ca}^{2+}$ refilling following depletion ${ }^{10,11}$.

Disruption to ER $\mathrm{Ca}^{2+}$, redox and ATP homoeostasis results in impaired protein folding and the accumulation of proteins in the $\mathrm{ER}^{12}$. Cell repair is promoted by ER stress signalling pathways, which alleviate the damage by inhibiting protein translation, upregulating protein 
folding and inducing protein degradation ${ }^{13}$. In addition, increased expression of SERCA enhances ER $\mathrm{Ca}^{2+}$ loading in response to both $\mathrm{Ca}^{2+}$ depletion from the ER and $\mathrm{Ca}^{2+}$-independent ER stress stimuli ${ }^{14-16}$. Multiple ER stress effectors also rapidly induce autophagy, which degrades protein aggregates and damaged organelles to aid cell survival ${ }^{17,18}$.

In the event of irreparable or prolonged damage, both the ER stress and autophagy pathways interact with those of apoptosis and cell fate is determined by the most dominant response ${ }^{19}$. In addition, $\mathrm{ER} \mathrm{Ca}^{2+}$ signalling at regions in close contact with mitochondria has a fundamental role in the regulation of cell fate; high levels of $\mathrm{Ca}^{2+}$ transfer results in the loss of mitochondrial integrity, thereby promoting caspase-dependent apoptosis ${ }^{20,21}$. Toxic levels of intracellular $\mathrm{Ca}^{2+}$ are provided by the action of ER and PM pumps/channels ${ }^{22-24}$. For example, altered SERCA activity mediates apoptosis via ER-mitochondrial $\mathrm{Ca}^{2+}$ transfer $^{25-28}$.

This study was initiated by the characterization by mass spectrometry of the protein-protein interactions of PERP required for apoptosis induction. We discovered that PERP is stabilized at the PM due to a reduction in its lysosomal uptake and degradation during ER stressinduced autophagy. Subsequently, there is an increased co-localization with the ER $\mathrm{Ca}^{2+}$ transporter SERCA2b that correlates with apoptosis induction. The findings thus identified a crosstalk between autophagy and apoptosis pathways with a role in the $\mathrm{Ca}^{2+}$-mediated regulation of cell fate at ER-PM junctions.

\section{Results}

\section{Identification of PERP-SERCA2b interaction and co- localization}

To identify novel effectors involved in PERP-mediated apoptosis, we investigated the protein-protein interactions of PERP using the HaloTag Mammalian Pull-Down System. A construct containing PERP cDNA fused to an $\mathrm{N}$-terminal HaloTag was generated and expressed in the human uveal melanoma cell line Mel202 where the fusion protein localized to the PM and secretory pathway (Fig. S1A), as previously characterized ${ }^{6}$. Expression of HaloPERP for $48 \mathrm{~h}$ significantly increased the protein levels of endogenous PERP and p53 and induced phosphatidylserine externalization (Fig. S1B and C), in line with previous results, showing that Halo-PERP functions in both p53 stabilization and apoptosis induction ${ }^{5}$. This data validated the HaloTag system as a suitable platform for characterization of the PERP protein interactome.

Halo-PERP protein pull-down and subsequent mass spectrometry analysis identified 21 proteins with a fold change of 1.5 or higher in at least two independent experiments and six proteins were identified exclusively in Halo-PERP pull-downs in all three experiments.
Interestingly, three ER membrane proteins were repeatedly identified in Halo-PERP pull-downs and were absent from all controls. Of these, we chose to focus on SERCA2 (Fig. 1a). The SERCA2 gene has three isoforms, SERCA2a-c, which differ in their C-terminal region and tissue-specific expression. SERCA2b is the house-keeping isoform, which has the highest affinity for $\mathrm{Ca}^{2+}$ and is expressed in all tissues ${ }^{10}$. As the peptide identified by mass spectrometry could not distinguish between the three isoforms, the specific interaction between PERP and SERCA2b was confirmed after HaloTag pull-down by Western blot using the isoform-specific antibody (Fig. 1b).

To further validate the interaction between PERP and SERCA2, HaloTag and Halo-PERP were expressed in Mel202 cells and SERCA2 interacting proteins were isolated by immunoprecipitation (IP). The specific isolation of Halo-PERP was confirmed by Western blot using a HaloTag antibody (Fig. 1c). Together these data identified and validated a consistent interaction between PERP and the ER $\mathrm{Ca}^{2+}$ pump SERCA2b (Fig. 1d).

We next aimed to characterize the localization of the PERP-SERCA2b interaction. To this end, mCherrySERCA2b was co-expressed in HeLa BAC Venus-PERP cells, which stably express Venus-PERP at physiological levels (Fig. 1e). Venus-PERP localized to the PM and secretory pathway and mCherry-SERCA2b localized broadly across the ER including the cortical ER, which lies adjacent to the PM. Notably, PERP was present in regions of the PM in close co-localization with the SERCA2bcontaining ER (shown by arrows).

The findings highlighted a novel interaction between the apoptosis effector protein PERP and the ER $\mathrm{Ca}^{2+}$ pump SERCA2b, which likely occurs at ER-PM points of contact.

\section{PERP protein is upregulated during ER stress independent of p53 transcriptional regulation}

Since SERCA2b is known to be induced in response to ER stress ${ }^{14}$ and we have revealed an interaction between PERP and SERCA2b, we next explored the possibility that PERP may also respond to ER stress. Brefeldin A (BFA) prevents the export of proteins from the Golgi apparatus, leading to the accumulation of proteins in the ER and activating ER stress response pathways. Prolonged exposure to BFA induces caspase-dependent apoptosis ${ }^{29}$. During conditions of ER stress, cells selectively transcribe genes with regulatory ER stress response elements in their promoters, including SERCA2 $\mathrm{b}^{14}$. BFA significantly increased the relative levels of SERCA2b mRNA from $2 \mathrm{~h}$ post-treatment (Fig. 2a), followed by an increase in SERCA2b protein levels from $8 \mathrm{~h}$ post-treatment (Fig. 2b).

HCT116 cells were used to study the response of endogenous PERP to BFA-induced ER stress and treatment with BFA for $8,16,24$ and $48 \mathrm{~h}$ significantly 


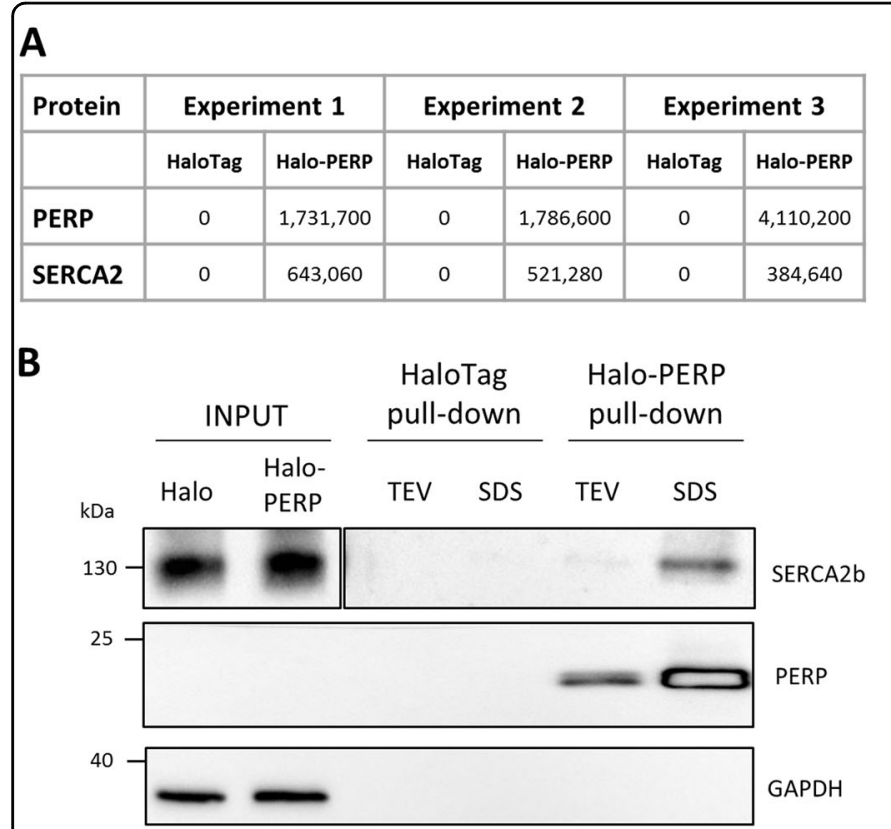

C

IP: SERCA2

Ab: HaloTag

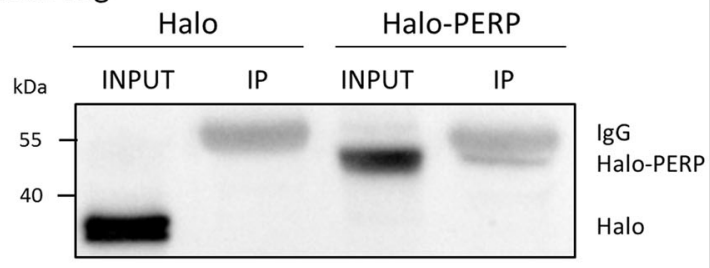

\section{E}
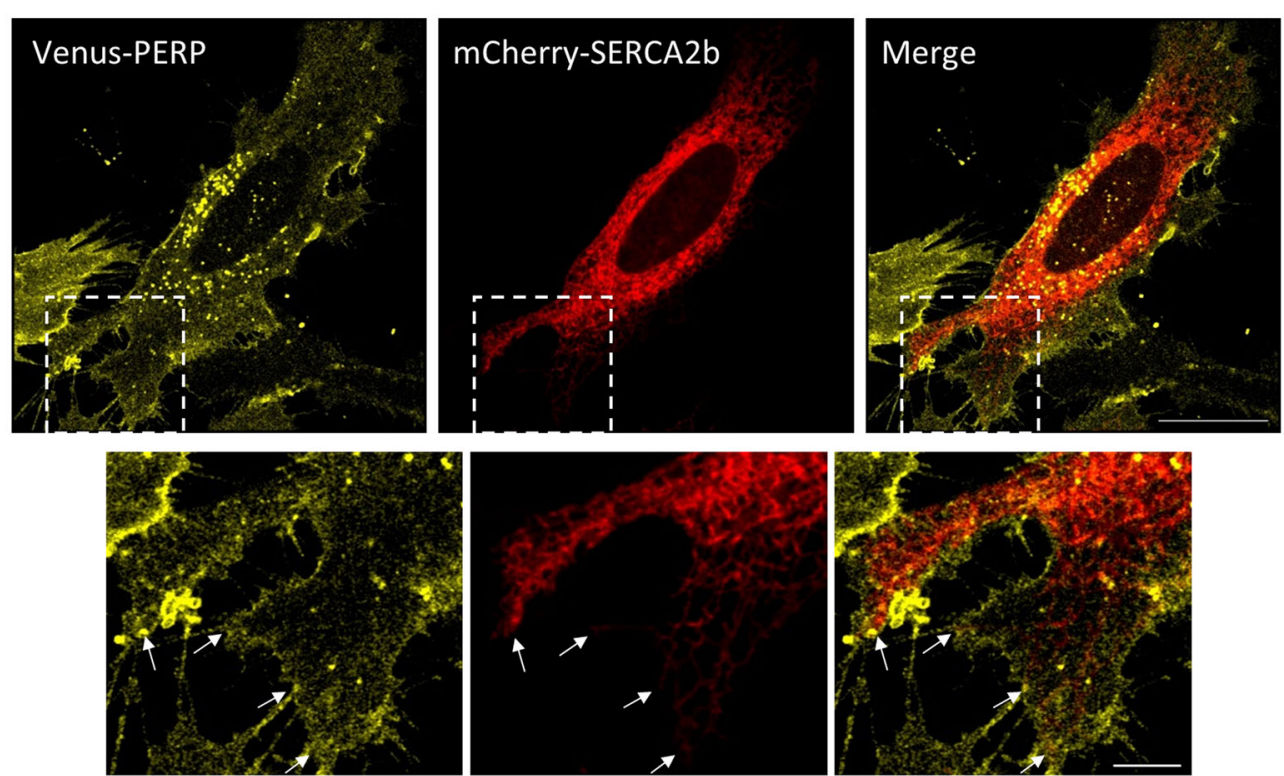

Fig. 1 PERP interacts with SERCA2b at ER-PM junctions. a Protein interacting partners of Halo-PERP were isolated from Mel202 cells using the HaloTag Mammalian Pull-Down System and identified by mass spectrometry. Maxquant intensity values for SERCA2 and PERP in three independent experiments are shown. $\mathbf{b}$ The interaction of PERP and SERCA2b was confirmed in two independent HaloTag pull-down experiments by

immunoblotting as described in the Methods (proteins eluted from the resin by TEV enzymatic cleavage followed by a successive SDS-based elution). Figure shows two different exposures of SERCA2b panel to allow visualization of lower intensity bands. c PERP-SERCA2 complex formation was validated by SERCA2 IP in Mel202 cells expressing HaloTag or Halo-PERP using a HaloTag antibody. $\mathbf{d}$ Diagram of SERCA2a-C proteins, indicating the relative positions of the peptide identified by mass spectrometry (black) and the antibody immunogen sites (grey) used for validation of the SERCA2b-PERP interaction. Antibody 1 was used in HaloTag pull down validation (b) and antibody 2 was used for IP of SERCA2 (c). e Super-resolution images of HeLa BAC Venus-PERP cells co-expressing mCherry-SERCA2b, junctions between the ER and PM shown by arrows. Scale bar: $20 \mu m$ in full image and $5 \mu \mathrm{m}$ in zoom panel

increased the protein levels of PERP (Fig. 2c). We next wanted to determine whether the increase in PERP during ER stress was due to an increase in transcription. HCT116 cells were treated with BFA and PERP mRNA levels were measured by RT-PCR over time. BFA-induced ER stress led to no significant increase in the mRNA levels of PERP, 


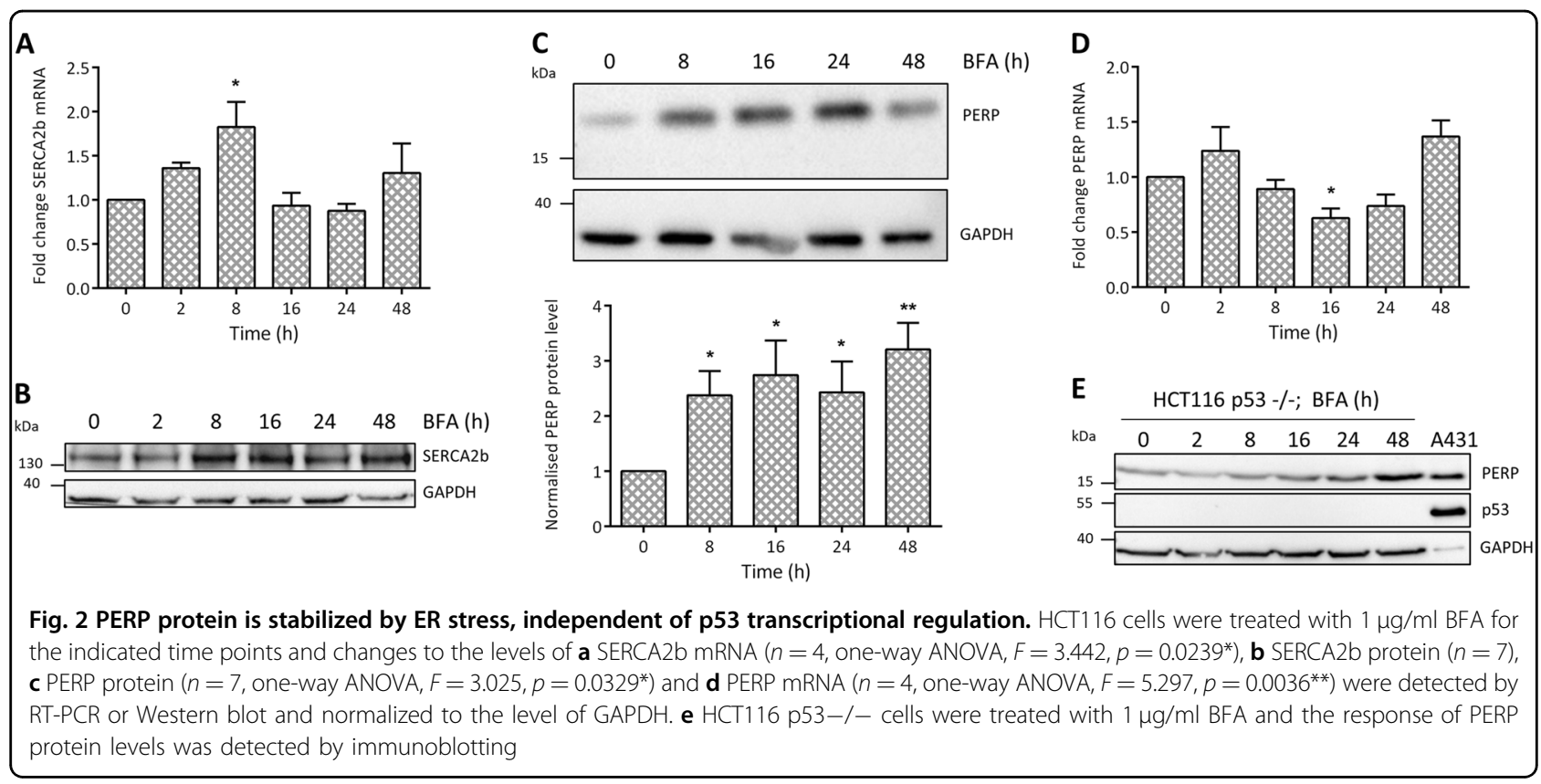

which actually significantly decreased at $16 \mathrm{~h}$ posttreatment (Fig. 2d). These findings were also consistent with those obtained in Mel202 cells (data not shown).

HCT116 p53-/- cells have significant deletions in exons $1-3$ of the p53 gene and produce p53 protein that is defective in its ability to bind to DNA ${ }^{30}$. Similarly to wildtype cells, HCT116 p53-/- cells upregulated PERP protein in response to prolonged ER stress (Fig. 2e), with no significant changes to PERP mRNA levels (data not shown). This finding suggested that p53 activity is not required for PERP to respond during conditions of ER stress. Furthermore, analysis of the PERP promoter region found no currently characterized ER stress response element in the first 1000 bases before exon one. This supported our finding that PERP is not transcriptionally regulated during conditions of ER stress.

In summary, the data presented here showed that although SERCA2b expression increased by transcription during conditions of ER stress, PERP protein levels increased via post-translational regulation in a p53independent manner.

\section{PERP accumulates at the PM in response to ER stress due to a reduction in its turnover}

PERP localization in response to ER stress was next assessed by super-resolution live-cell microscopy. VenusPERP localized non-homogenously across the PM of nontreated cells and its levels were highest in regions of the membrane that formed contacts with neighbouring cells (Fig. 3a). Treatment with BFA for $16 \mathrm{~h}$ induced the accumulation of Venus-PERP across the cell surface and within ER tubules, with the formation of ER vacuoles due
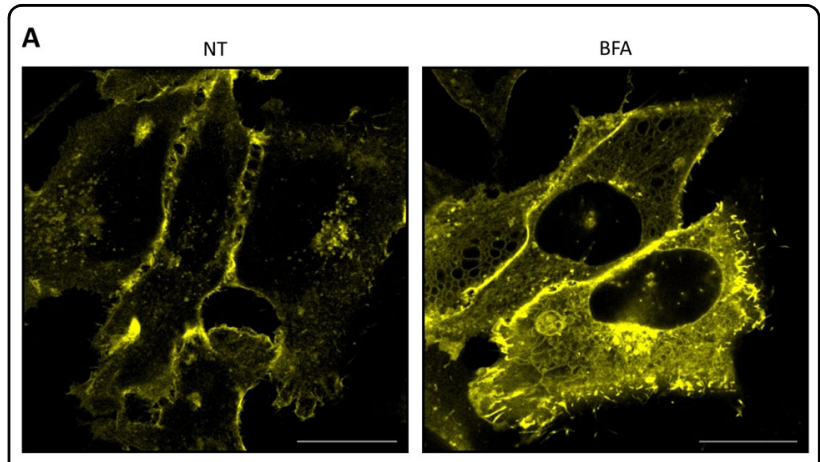

B

NT

BFA
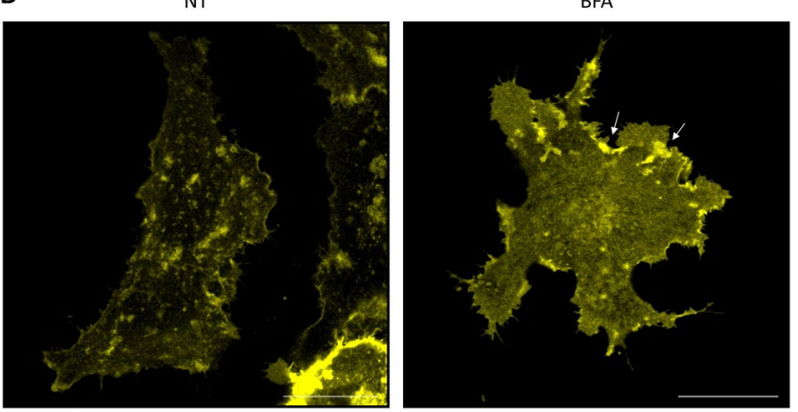

Fig. 3 PERP accumulates at plasma membrane puncta during ER stress. $\mathbf{a}$, b Super-resolution images of HeLa BAC Venus-PERP cells at the $z$-stack centre (a) and base of the cell (b), either non-treated (NT) or BFA-treated (16 h). Scale bars: $20 \mu \mathrm{m}$

to the prolonged induction of ER stress. The accumulation of ER-localized Venus-PERP was likely due to the BFA-induced inhibition of Golgi-PM trafficking and the retrograde transport of Golgi proteins into the ER. This 
finding confirmed that PERP reaches the PM via the classical secretory pathway.

The distribution of PERP at the PM during conditions of ER stress was analysed at the adherent base of the cell (Fig. 3b). Venus-PERP localized to distinct PM islands in non-treated cells and significant areas of the membrane were PERP protein-free. In response to BFA treatment, Venus-PERP displayed a more homogeneous distribution across the PM and formed intense puncta at the cell periphery (shown by arrows). Significantly, since BFA inhibits Golgi-PM trafficking, the accumulation of Venus-PERP at the PM was not due to the delivery of newly synthesized protein. Therefore, the increase in the PM levels of PERP under ER stress conditions was likely due to a reduction in its translocation from the PM and in its subsequent degradation.
Together these findings indicated that PERP accumulates at the PM during ER stress via an increase in its protein stability and a reduction in its turnover.

\section{PERP protein is actively degraded by the lysosome}

We therefore next characterized the stability and degradation of PERP protein. HCT116 cells were treated with the protein synthesis inhibitor cycloheximide (CHX) and the protein levels of PERP were detected over time (Fig. 4a). p53, used as a positive control for protein synthesis inhibition, decreased from $2 \mathrm{~h}$ post-treatment due to its short half-life. The protein levels of PERP remained constant at $2 \mathrm{~h}$ post-CHX treatment followed by a significant decrease from $4 \mathrm{~h}$ post-treatment. Remarkably, PERP protein levels were reduced by $50 \%$ after $4 \mathrm{~h}$ of protein synthesis inhibition.
A
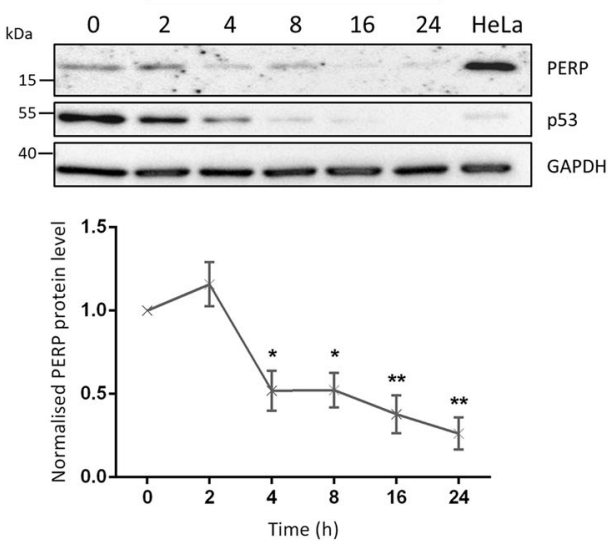

B

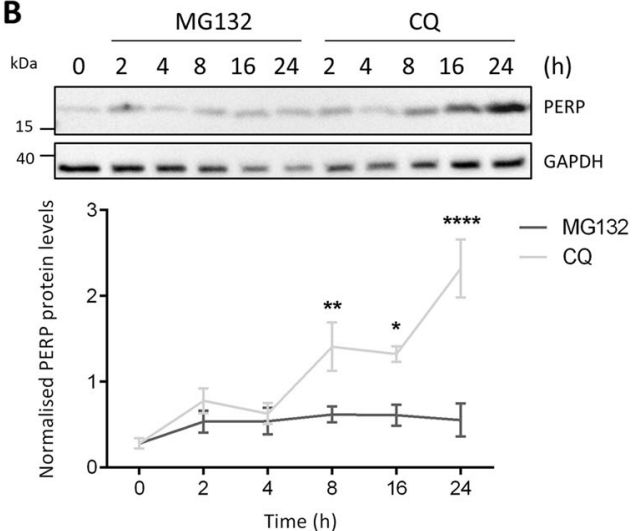

C
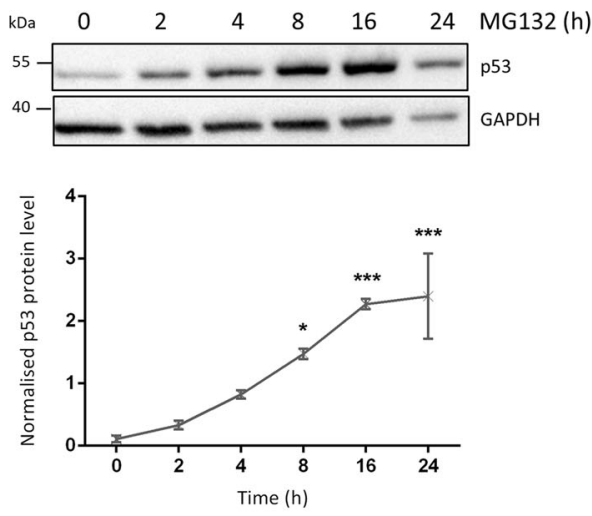

D

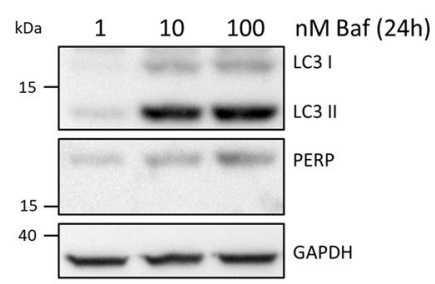

Fig. 4 PERP protein is actively degraded by the lysosome. a HCT116 cells were treated with $30 \mu \mathrm{g} / \mathrm{ml} \mathrm{CHX} \mathrm{for} \mathrm{the} \mathrm{indicated} \mathrm{time} \mathrm{points,} \mathrm{and} \mathrm{the}$ protein levels of PERP and $p 53$ were detected by immunoblotting. Histogram shows PERP protein levels normalized to GAPDH. One-way ANOVA, $n$ $=3, F=12.63, p=0.0003^{* * *}$. b HCT116 cells were treated with $20 \mu \mathrm{M} \mathrm{MG132}$ or $100 \mu \mathrm{M}$ CQ for the indicated time points and PERP protein levels were detected by Western blot. Graph represents PERP protein levels normalized to GAPDH. One-way ANOVA, $n=3, M G 132$ PERP: $F=0.8849, p=$ 0.5204 ns; CQ PERP: $F=13.03, p=0.0002^{* * *}$. c HCT116 cells were treated with $20 \mu \mathrm{M}$ MG132 and the protein levels of p53 were detected by immunoblotting and normalized to the level of GAPDH. One-way ANOVA, $n=3, F=11.58, p=0.0003^{* * *}$. $\mathbf{d}$ HCT116 cells were treated with 1 , 10 and $100 \mathrm{nM}$ of Baf and the protein levels of LC3B and PERP were detected $24 \mathrm{~h}$ post-treatment by immunoblotting 


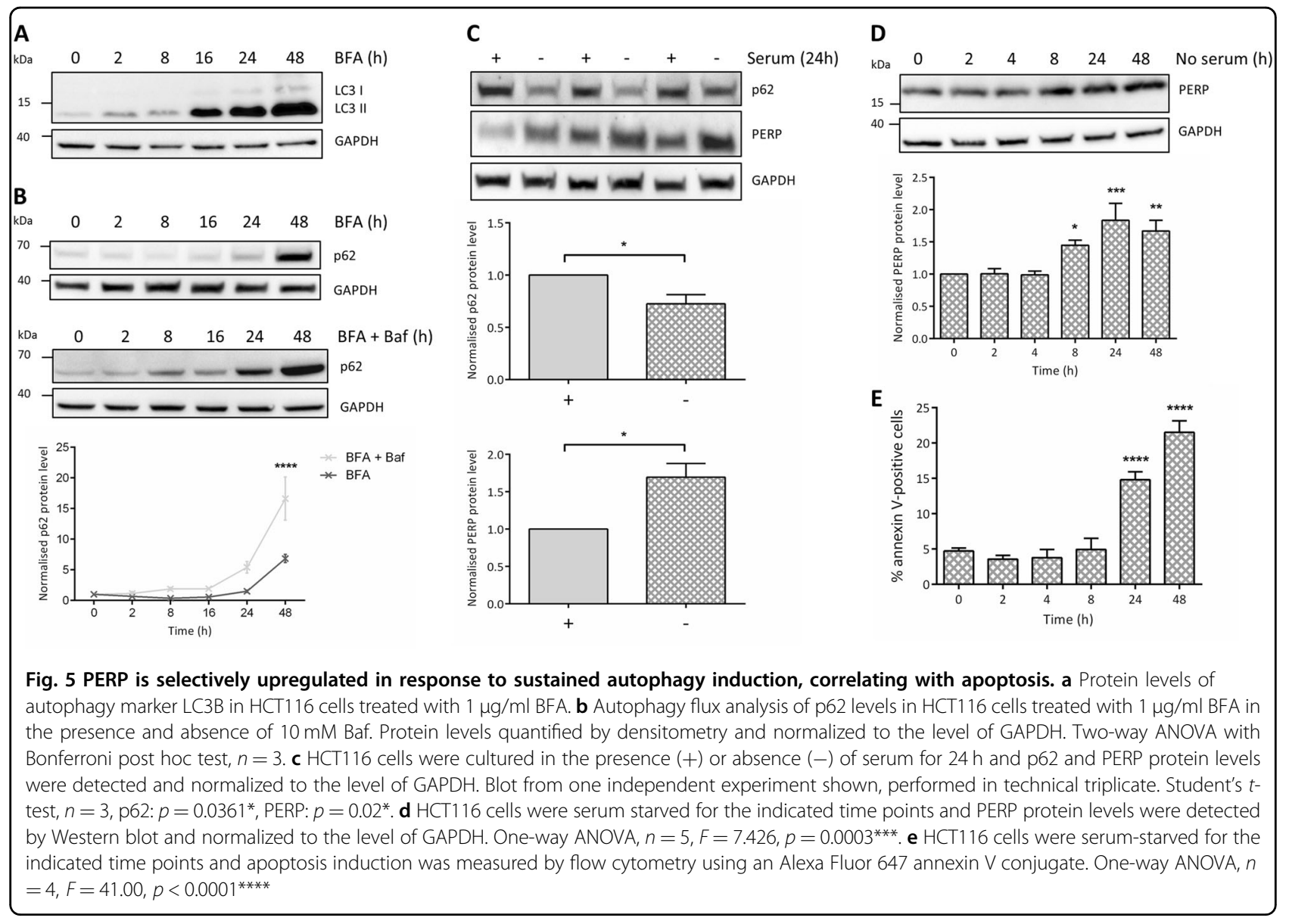

We also characterized the pathway that mediates the degradation of PERP protein. HCT116 cells were treated with the proteasome inhibitor MG132 or the lysosome inhibitor chloroquine (CQ) and cell lysates were collected for up to $24 \mathrm{~h}$ (Fig. $4 \mathrm{~b}$ ). Inhibition of the proteasome degradation pathway led to no significant changes to PERP protein levels. However, PERP protein levels progressively increased from $2 \mathrm{~h}$ of lysosome inhibition, with a significant increase from $8 \mathrm{~h}$ post-treatment. This suggested that PERP protein undergoes lysosomal degradation. The protein levels of $\mathrm{p} 53$ were used as a positive control for proteasome inhibition by MG132; p53 increased from $2 \mathrm{~h}$ post-MG132 treatment with a significant increase from $8 \mathrm{~h}$ post-treatment (Fig. 4c). PERP lysosomal degradation was confirmed using a second lysosome inhibitor bafilomycin (Baf) and the protein levels of both PERP and the autophagy marker LC3II increased in a dose-dependent manner $24 \mathrm{~h}$ after treatment (Fig. 4d).

Together this data showed that PERP protein is actively turned-over in healthy cells by a pathway requiring lysosomal function.

\section{Upregulation of PERP by sustained autophagy precedes apoptosis induction}

Multiple ER stress signalling pathways regulate autophagy and so we next characterized the response of autophagy markers LC3B and p62 to BFA (Fig. 5a, b). BFA consistently increased the levels of LC3II from $2 \mathrm{~h}$ of ER stress induction, and at 16,24 and $48 \mathrm{~h}$ post-treatment, LC3II increased beyond the dynamic range required for quantification compared to the non-treated sample. This confirmed that the number of autophagosomes progressively increased over time in response to BFA-induced ER stress.

The protein levels of the autophagy marker p62 were used for autophagy flux analysis. BFA decreased the levels of p62 at 2 and $8 \mathrm{~h}$, followed by an increase until a peak level at $48 \mathrm{~h}$ post-treatment. To determine whether the changes to LC3II and p62 protein levels in response to BFA were due to an increase in autophagy induction or an impairment in lysosomal degradation, HCT116 cells were pre-treated with Baf followed by BFA for the indicated time points (Fig. 5b). Normalized p62 levels increased beyond that of the BFA-only-treated cells at all time 


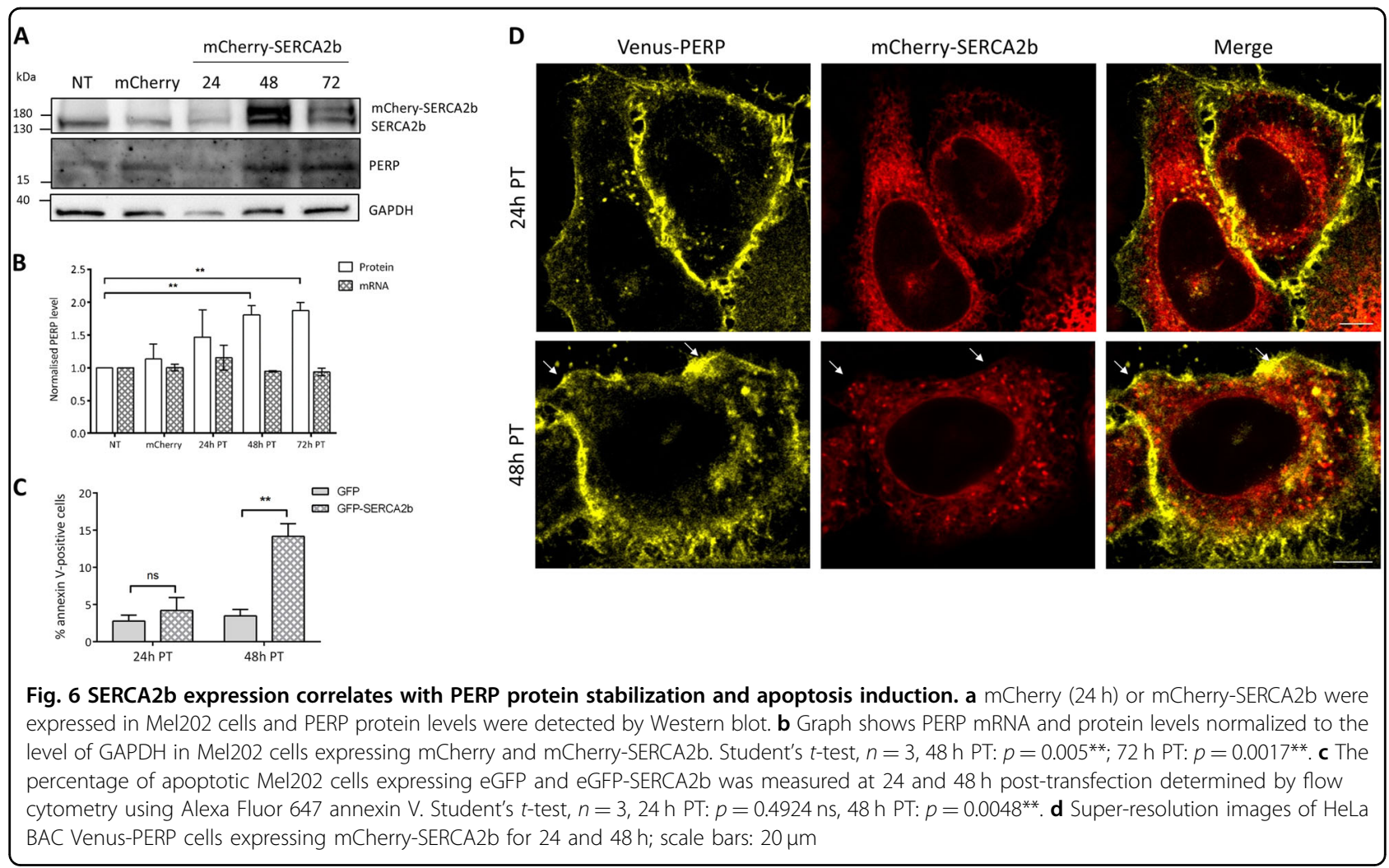

points tested, which suggested that the increase in LC3II and p62 in response to BFA was due to an increase in autophagy induction rather than lysosomal inhibition.

We next determined the effect of autophagy induction on the protein levels of PERP. Firstly, HCT116 cells were serum starved for $24 \mathrm{~h}$ and the protein levels of autophagy marker p62 and PERP were determined (Fig. 5c); p62 was significantly decreased confirming autophagy induction as characterized in this cell type elsewhere ${ }^{31}$. In addition, PERP protein levels were significantly increased suggesting that PERP responds to autophagy induction.

We therefore next characterized the response of PERP to autophagy induction over time. HCT116 cells were serum starved for $2-48 \mathrm{~h}$ and the response of PERP was detected by immunoblotting. PERP protein levels remained constant for up to $4 \mathrm{~h}$ of serum starvation but significantly increased at 8,24 and $48 \mathrm{~h}$ post-autophagy induction (Fig. 5d). The sustained activation of autophagy promotes cell death and so the impact of serum starvation on apoptosis was determined over time by annexin $\mathrm{V}$ staining and flow cytometry; apoptosis was significantly induced from $24 \mathrm{~h}$ of serum starvation (Fig. 5e).

Together our findings showed that during prolonged autophagy induction, such as that mediated by ER stress or serum starvation, PERP protein is upregulated before the switch to apoptosis induction.
Increased SERCA2b leads to stabilization of PERP protein and induces apoptosis

Increased levels of SERCA2 are known to induce caspase-dependent apoptosis due to $\mathrm{ER} \mathrm{Ca}^{2+}$ overload $^{25,26}$. To determine whether PERP is involved in SERCA2b-mediated apoptosis, mCherry-SERCA2b was expressed in Mel202 cells and changes to PERP protein and mRNA levels were detected (Fig. 6a, b). At $24 \mathrm{~h}$ posttransfection, mCherry-SERCA2b was expressed at low levels and no changes to the mRNA or protein levels of PERP were observed. However, at 48 and $72 \mathrm{~h}$ posttransfection, both endogenous SERCA2b and mCherrySERCA2b were increased compared to the control cells and this correlated with a significant increase in the protein levels of PERP. Despite this, expression of mCherry-SERCA2b for up to $72 \mathrm{~h}$ had no effect on the mRNA levels of PERP (Fig. 6b). This suggested that increased expression of SERCA2b stabilizes PERP protein.

The SERCA2b-mediated apoptosis in Mel202 cells was determined at both 24 and $48 \mathrm{~h}$ post-transfection by flow cytometry using annexin V (Fig. 6c). At $24 \mathrm{~h}$ posttransfection with eGFP-SERCA2b, there was no significant difference in the level of apoptosis compared to the eGFP expressing control cells. However, apoptosis was significantly induced at $48 \mathrm{~h}$ post-transfection with eGFP-SERCA2b. This showed that increased SERCA2b 

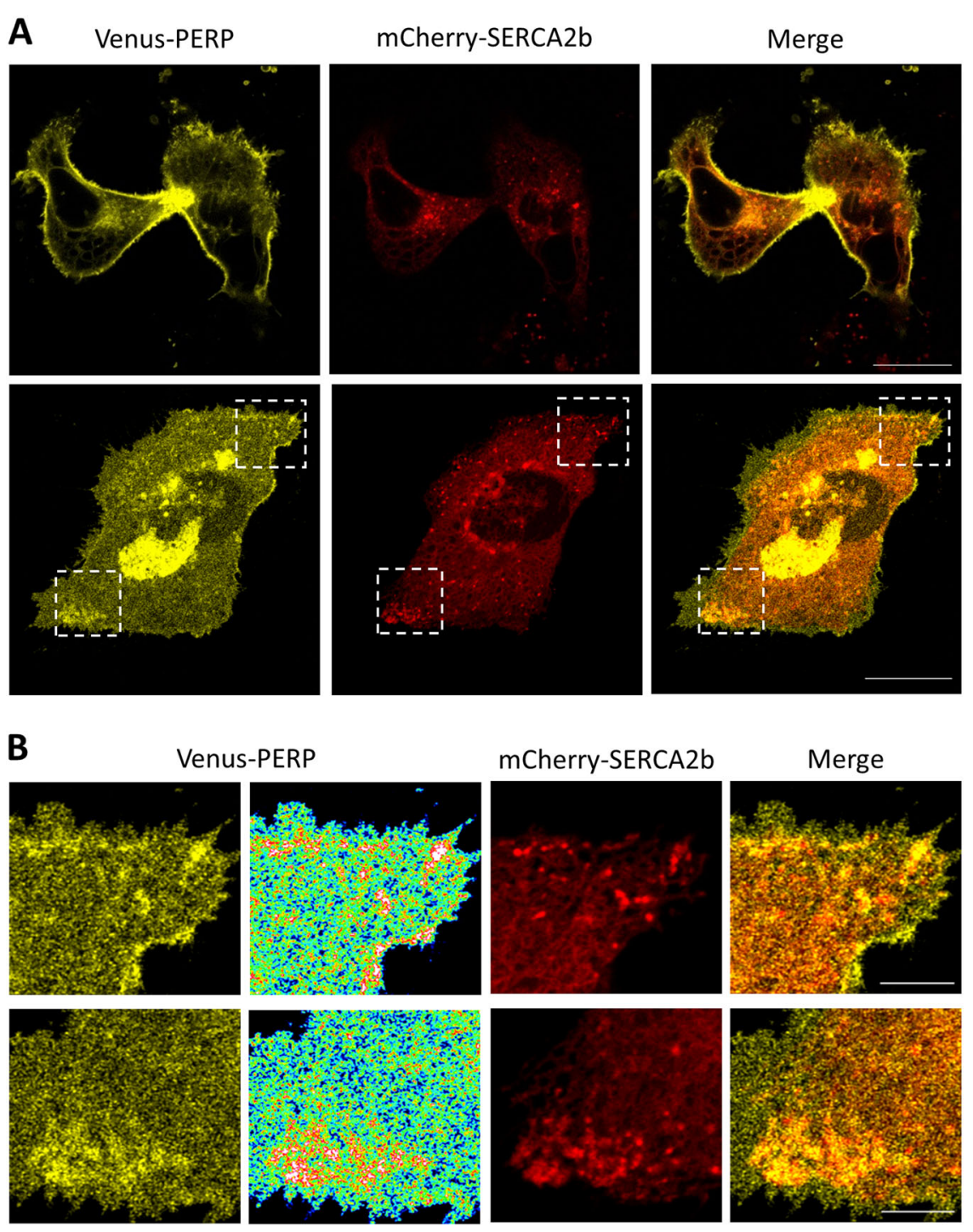

Fig. 7 PERP increasingly co-localizes with SERCA2b during ER stress. a HeLa BAC Venus-PERP cells co-expressing mCherry-SERCA2b were treated with $1 \mu \mathrm{g} / \mathrm{ml} \mathrm{BFA}$ for $16 \mathrm{~h}$ and co-localization was assessed at the mid and bottom optical sections. Scale bars: $20 \mu \mathrm{m}$. $\mathbf{b}$ Enlargement of boxed areas with rainbow pseudo colour-coded distribution of Venus-PERP. Scale bars: $5 \mu \mathrm{m}$

expression beyond the homoeostatic threshold level at $48 \mathrm{~h}$ post-transfection mediated apoptosis. Furthermore, PERP was stabilized specifically during SERCA2bmediated apoptosis.

Next, mCherry-SERCA2b was co-expressed in HeLa BAC Venus-PERP cells and images were taken at 24 and $48 \mathrm{~h}$ post-transfection (Fig. $6 \mathrm{~d}$ ). At $24 \mathrm{~h}$ post-transfection, the mCherry-SERCA2b-containing ER formed healthy tubules throughout the cell and subplasmalemmal region. However, at $48 \mathrm{~h}$ post-transfection, mCherry-SERCA2b accumulated in intense puncta throughout the ER and cortical ER. Most notably, Venus-PERP was present in the regions of the PM that co-localized with the mCherrySERCA2b puncta (shown by arrows in Fig. 6d).
Together, this data indicated that PERP protein is stabilized in the presence of apoptosis-inducing levels of SERCA2b expression.

\section{PERP and SERCA2b accumulate at ER-PM junctions in response to ER stress}

Next, the effect of sustained ER stress on the colocalization of PERP and SERCA2b was determined. HeLa BAC Venus-PERP cells co-expressing mCherry-SERCA2b were treated with BFA and images were taken $16 \mathrm{~h}$ posttreatment (Fig. 7a). Imaging in the centre of the optical $z$ stack of the cell showed co-distribution of Venus-PERP and mCherry-SERCA2b in the ER and vacuoles formed across the ER network. In addition, high levels of PERP 
across the cell surface were observed. Since the accumulation of PERP in the ER was potentially due to an indirect effect of the mechanism of BFA, the co-localization of Venus-PERP and mCherry-SERCA2b was determined at the base of the cell. Interestingly, mCherry-SERCA2b accumulated in intense puncta towards the PM in regions where Venus-PERP was also enriched (Fig. 7b).

Taken together, the findings indicate that during conditions of sustained ER stress, PERP accumulates at the PM due to a reduction in its lysosomal uptake and degradation, where it increasingly co-localizes with SERCA2b across ER-PM points of contact to mediate apoptosis.

\section{Discussion}

The protein levels of the p53-regulated PM protein PERP are maintained at a threshold level in healthy cells ${ }^{6}$. In this study, we showed that the threshold level of PERP protein is regulated through the action of a selective autophagy-lysosomal pathway. Therefore, PERP expression is precisely controlled by competing mechanisms that are sensitive to cellular state including, but not limited to, p53/p63-mediated transcription and autophagy-lysosomal protein degradation.

Using a cell-based model expressing physiological levels of PERP, we showed that PERP has a heterogeneous distribution across the PM of healthy cells with highest expression in regions of the PM that are engaged in cell-cell contacts. These areas are likely to be important for the cell adhesion function of PERP ${ }^{3,32}$. In addition, the identification of an interaction between PERP and the ER-PM junctional protein SERCA2b highlighted, for the first time, localization of PERP to regions of the PM that come in close contact with the ER.

Expression of PERP above a threshold level influences cell fate towards the death outcome $e^{1,5-7}$. Here, we showed that sustained autophagy, induced by both starvation and ER stress, increased PERP protein at the PM beyond the physiological threshold level and this correlated with apoptosis induction. Autophagy is also induced following Salmonella infection to aid pathogen clearance $^{33,34}$. Recently it was shown that PERP accumulates at the apical PM in response to Salmonella infection due to alterations in its uptake and degradation ${ }^{35}$. We therefore propose that the upregulation of autophagy following infection promotes the stabilization of PERP at the PM.

Complex interactions between autophagy and apoptosis following cellular disturbances, such as ER stress, enable cells to dynamically regulate cell fate in a highly controlled manner ${ }^{36}$. Our findings suggest that PERP is involved in the autophagy/apoptosis crosstalk; PERP is selectively upregulated at the PM following high levels of autophagy (starvation, ER stress, inflammation) where it directly engages its apoptotic machinery. In this scenario, PERP is protective against chronic autophagy.

Signals transduced across membrane contact sites via the formation of protein complexes and the transfer of molecules, such as $\mathrm{Ca}^{2+}$, regulate many cellular processes $^{37}$. SERCA2b is recruited to ER-PM junctions involved in SOCE where it is key to establishing $\mathrm{Ca}^{2+}$ homoeostasis after oscillation ${ }^{11}$. PERP lacks a conserved death domain and its precise mode of apoptosis induction from the PM is not understood ${ }^{7}$. Here, we found that PERP is post-transcriptionally upregulated during SERCA2b-mediated apoptosis, conceivably through ER stress induced by dysregulation of luminal $\mathrm{Ca}^{2+}$ homoeostasis $^{12,25}$. In addition, PERP and SERCA2b increasingly co-localize during chronic ER stress. PERP is the first identified PM-localized interactor of SERCA2b and we therefore propose that this interaction promotes the stabilization of SERCA2b in the cortical ER for sustained $\mathrm{Ca}^{2+}$ signalling events.

SERCA modulates the sensitivity to apoptosis and its $\mathrm{Ca}^{2+}$ pumping activity is regulated by competing proapoptotic and anti-apoptotic pathways ${ }^{38-41}$. Apoptosis modulators, such as p53, activate SERCA2 to promote $\mathrm{Ca}^{2+}$-dependent apoptosis ${ }^{39}$. Similarly, the PERP-SERCA2b interaction may mediate apoptosis by mitochondrial $\mathrm{Ca}^{2+}$ overload. This is supported by a study which showed that PERP induces apoptosis via an increase in mitochondrial membrane permeability and the release of cytochrome $\mathrm{C}$ in renal cells exposed to hypoxic injury ${ }^{42}$.

Our current findings provide the first mechanistic evidence of SERCA2 regulation and apoptosis induction at ER-PM junctions. The interaction of PERP and SERCA2b at junctions involved in SOCE may promote the sustained delivery of toxic levels of $\mathrm{Ca}^{2+}$ to the ER. However, PERP has a high sequence similarity with established $\mathrm{Ca}^{2+}$ channels and so it remains possible that PERP has $\mathrm{Ca}^{2+}$ conducting activity across the $\mathrm{PM}^{1}$. The interaction between PERP and SERCA2b would therefore directly deliver extracellular $\mathrm{Ca}^{2+}$ into the ER for apoptosis.

This study has identified a novel crosstalk between the ER stress, autophagy and apoptosis pathways and has highlighted, for the first time, a mechanism of apoptosis regulation at ER-PM junctions. PERP-mediated destabilization of $\mathrm{ER} \mathrm{Ca}^{2+}$ metabolism is likely to further induce both ER stress and autophagy responses and therefore amplify the stress signal to sway cell fate towards apoptosis.

\section{Materials and methods \\ Cell culture}

Authenticated Mel202 cells were purchased from Public Health England (lot number 13H016) and were cultured in RPMI 1640 with $2 \mathrm{mM}$ L-glutamine and $25 \mathrm{mM}$ HEPES (Gibco, Life Technologies, Paisley, UK) supplemented 
with 10\% FCS (Sigma-Aldrich, Dorset, UK), $1 \mathrm{mM}$ sodium pyruvate and $1 \%$ non-essential amino acids (Sigma-Aldrich). HCT116 and HCT116 p53-/- cells (obtained from Johns Hopkins University GCRF Core Cell Center, Baltimore, USA; HCT116 p53+/+ (parent of p53 KO), lot 40-16; HCT116 p53-/-, lot 379.2) were grown in Modified McCoy's 5a medium (Gibco) supplemented with $10 \%$ FCS. HeLa cells (original lot purchased from ATCC, catalogue number ATCC CCL-2) stably expressing Venus-PERP from a bacterial artificial chromosome (HeLa BAC Venus-PERP cells, Raheela Awais, personal communication) were cultured in Eagle's Minimum Essential Medium (ATCC, Middlesex, UK) supplemented with $10 \%$ FCS and $0.8 \mathrm{mg} / \mathrm{ml}$ geneticin (Sigma-Aldrich). All cells were cultured at $37^{\circ} \mathrm{C}$ and $5 \%$ $\mathrm{CO}_{2}$ in humidified incubators and were free of mycoplasma contamination.

\section{Expression vectors}

GFP-PERP, eGFP-SERCA2b and mCherry-SERCA2b were described previously ${ }^{6,11}$. To generate the HaloPERP construct, PERP cDNA was amplified by PCR from the GFP-PERP vector using primers designed to add flanking EcoRI and NotI restriction sites to the $5^{\prime}$ and $3^{\prime}$ regions, respectively, for subsequent incorporation into the pHTN HaloTag vector (Promega, Southampton, UK). The primer sequences were: forward primer $\left(5^{\prime}\right.$-AAT TAGAATTCATGATCCGCTGCGGCCTG-3') and reverse primer (5'-AATTAGCGGCCGCTTAGGCA GATGTGTAGAAGTACCTGGG-3'), with restriction sites shown in bold and stop codon underlined. PCR amplification was performed using the Phusion HighFidelity PCR Kit (New England Biolabs Inc, Ipswich, UK) and the final reaction contained $250 \mathrm{ng}$ of template DNA, $0.5 \mu \mathrm{M}$ forward and reverse primers, $200 \mu \mathrm{M}$ dNTPs, $1 \times$ HF buffer and 1 unit of T4 DNA polymerase. Cycling conditions used were 1 cycle of $98^{\circ} \mathrm{C}$ for $30 \mathrm{~s}$, followed by 30 cycles of $98^{\circ} \mathrm{C}$ for $10 \mathrm{~s}, 63^{\circ} \mathrm{C}$ for $30 \mathrm{~s}$ and $72^{\circ} \mathrm{C}$ for $30 \mathrm{~s}$, terminated with one cycle of $72^{\circ} \mathrm{C}$ for $7 \mathrm{~min}$.

Amplified DNA was purified using the QIAquick PCR Purification Kit (Qiagen Ltd, Manchester, UK) and both the PERP sequence and HaloTag vector were digested using EcoRI and NotI (Roche, West Sussex, UK). Ligation was performed using 1 unit of T4 DNA ligase, $1 \times$ ligation buffer, $80 \mathrm{ng}$ of backbone DNA and $35 \mathrm{ng}$ of insert DNA. The resulting vector was used to transform $\mathrm{DH} 5 \alpha$ Escherichia coli (Invitrogen, Paisley, UK), selected using ampicillin $(100 \mu \mathrm{g} / \mathrm{ml})$ and purified using the Qiagen EndoFree Plasmid Maxi Kit. The sequence of HaloTagPERP (Halo-PERP) was confirmed by DNA sequencing, performed by DNA Sequencing \& Services (School of Life Sciences, University of Dundee, Scotland) on an Applied Biosystems model 3730 automated capillary DNA sequencer.

\section{Cell transfection and treatment}

Mel202 cells were transiently transfected with HaloPERP, mCherry-SERCA2b and control vectors. Cells were seeded at a density of $4 \times 10^{5}$ cells/well in 6-well plates and the following day were transfected with $1 \mu \mathrm{g}$ of plasmid DNA and $2 \mu \mathrm{l}$ of TurboFect in vitro transfection reagent (Thermo Scientific, Paisley, UK). For live-cell imaging, $3 \times 10^{5} \mathrm{HeLa}$ BAC Venus-PERP cells were seeded in 35-mm glass-bottom dishes (Greiner Bio-One, Stonehouse, UK) and transfected with $1 \mu \mathrm{g}$ of mCherrySERCA2b using $2 \mu \mathrm{l}$ of TurboFect. For HaloTag pulldown, $2.5 \times 10^{6}$ cells were seeded in 100-mm dishes (four dishes per transfection) and transfected using $5 \mu \mathrm{g}$ of plasmid DNA and $10 \mu \mathrm{l}$ of TurboFect. Cells were treated with $1 \mu \mathrm{g} / \mathrm{ml}$ BFA, $20 \mu \mathrm{M}$ MG132, $100 \mu \mathrm{M}$ CQ, or 1,10 and $100 \mathrm{nM}$ Baf A1 (all from Sigma-Aldrich).

\section{HaloTag protein pull-down}

PERP protein-protein interactions were isolated using the HaloTag Mammalian Pull-Down System (Promega) and standard protocol. Briefly, $1 \times 10^{7} \mathrm{Mel} 202$ cells were transfected with either HaloTag or Halo-PERP plasmids and cell lysates were collected $24 \mathrm{~h}$ post-transfection. A total of $2 \mathrm{mg}$ of each protein lysate was incubated with pre-washed HaloLink resin at $4{ }^{\circ} \mathrm{C}$ overnight. The resin was washed five times using TBS with 0.05\% IGEPAL CA360 (Sigma-Aldrich) and PERP, plus any interacting proteins were cleaved from the resin using 30 units of ProTEV enzyme (Promega) at room temperature for $1 \mathrm{~h}$ (elution 1). Following removal of the protein-containing supernatant, the resin was heated to $95^{\circ} \mathrm{C}$ for $5 \mathrm{~min}$ in SDS sample buffer (elution 2). Proteins in both elutions were identified by mass spectrometry and independently confirmed by Western blot.

\section{Immunoprecipitation}

SERCA2 was immunoprecipitated using the Dynabeads Protein A Immunoprecipitation Kit (Invitrogen) and monoclonal SERCA2 antibody (1:100, ab2861 Abcam, Cambridge, UK). Cells were scraped in dPBS, pelleted and frozen to $-80^{\circ} \mathrm{C}$. The resulting pellet was lysed in Mammalian Lysis Buffer with Protease Inhibitor Cocktail (both from Promega) and $1.5 \mathrm{mg}$ of protein was incubated with the bead-antibody conjugate for $30 \mathrm{~min}$ at room temperature. Antibody-protein complexes were washed four times in PBS and proteins were removed in SDS sample buffer for Western blot analysis.

\section{In-gel digestion and mass spectrometry}

For mass spectrometry analysis, proteins were separated on NuPAGE 4-12\% Bis-Tris gradient gels (Invitrogen) and each lane was cut into three slices. Samples were reduced in $10 \mathrm{mM}$ dithiothreitol at $56^{\circ} \mathrm{C}$, followed by alkylation using $50 \mathrm{mM}$ iodoacetamide at room 
temperature for $30 \mathrm{~min}$. Proteins were digested using $0.08 \mu \mathrm{g}$ of Trypsin Gold (Promega) per gel slice at $37^{\circ} \mathrm{C}$ for $16 \mathrm{~h}$ and peptides were extracted from the band pieces using acetonitrile, dried in a SpeedVac and re-suspended in $1 \%$ formic acid.

A total of $5 \mu \mathrm{l}$ of peptides were separated on a nanoACQUITY UPLC system (Waters, Herts, UK), followed by a LTQ Orbitrap XL mass spectrometer (Thermo Scientific) with a Proxeon nanoelectrospray source. Peptides were passed through a $5 \mathrm{~cm} \times 180 \mu \mathrm{m}$ BEH-C18 symmetry trapping column, followed by a $25 \mathrm{~cm} \times 75 \mu \mathrm{m}$ BEH-C18 column (both from Waters) at a flow rate of $400 \mathrm{nl} / \mathrm{min}$ for $39 \mathrm{~min}$. The mass spectrometer acquired full scan MS spectra $(m / z 300-2000$, resolution 30,000) and the five most abundant ions were further fragmented for MS/MS analysis in the LTQ. A blank of $1 \%$ formic acid was ran between each sample. Raw mass spectrometry files were analysed using Maxquant proteomics software (version 1.5.3.30) and proteins were identified using the whole HumanIPI database (June 2016).

\section{Super-resolution live-cell microscopy}

Super-resolution imaging was performed using a Zeiss LSM 880 Axio Observer microscope with Airyscan detection system in SR mode using a Plan-Apochromat $63 \times / 1.4$ Oil DIC M27 objective (Carl Zeiss, Jena, Germany). Excitation was achieved using the $488 \mathrm{~nm}$ line from an argon laser and a $561 \mathrm{~nm}$ diode laser or $594 \mathrm{~nm}$ $\mathrm{HeNe}$ laser and emitted light was collected through appropriate filters to eliminate any spill-over. Raw images were immediately Airyscan processed using the default settings and analysed using ZEN 2.1 software.

\section{Fluorescent microscopy}

Halo-PERP was labelled with the TMRDirect HaloTag ligand and $24 \mathrm{~h}$ post-transfection cells were imaged using a Zeiss Axio Observer Z1 live-cell microscope with Apotome.2 system and a 40×/1.3 Oil DIC objective (Carl Zeiss).

\section{Western blotting and antibodies}

Cells were harvested following transfection or treatment at specific time points in lysis buffer $(0.128 \mathrm{M}$ $\beta$-mercaptoethanol, $40 \mathrm{mM}$ tris, $10 \%$ glycerol, $1 \%$ SDS and $0.01 \%$ bromophenol blue) containing PhosSTOP phosphatase and Complete protease inhibitor cocktails (both from Roche). Proteins were separated by SDS-PAGE on a $10 \%$ polyacrylamide gel, transferred to nitrocellulose membrane and probed with the primary antibody at $4{ }^{\circ} \mathrm{C}$ overnight. Antibodies for PERP (ab5986), SERCA2b isoform specific (ab137020) and GAPDH (ab8245) were purchased from Abcam, and antibodies for LC3B (\#3868) and p62 (\#8025) were from Cell Signalling Technology (Leiden, The Netherlands). Anti-HaloTag (G9211) was from Promega and anti-p53 (P6874) was from SigmaAldrich. Immunocomplexes were incubated with the appropriate horseradish peroxidase-conjugated secondary antibody and detected using SuperSignal West Pico Chemiluminescent Substrate (Thermo Scientific) or Radiance Plus Chemiluminescent Substrate (Cambridge Bioscience, Cambridge, UK) and a Bio-Rad Chemidoc Imaging System. Membranes were incubated in stripping solution at $55^{\circ} \mathrm{C}$ for $25 \mathrm{~min}$ and sequentially re-probed.

\section{RNA extraction, reverse transcription and RT-PCR}

RNA was extracted using an RNeasy Mini Kit (Qiagen) and converted to cDNA using the First Strand cDNA Synthesis Kit (Thermo Scientific). Real-time quantitative PCR was performed for both PERP and GAPDH using the primers and protocol described previously ${ }^{43}$. Primers were designed to specifically amplify the full-length SERCA2 transcript (SERCA2b) at an annealing temperature of $60{ }^{\circ} \mathrm{C}$ (forward primer $5^{\prime}$-TCGAACCCTTGC CACTCATC- $3^{\prime}$ and reverse primer 5'-GCACAAACG GCCAGGAAATC-3', synthesized by Eurogentec, Southampton, UK).

\section{Flow cytometry apoptosis detection}

Floating and attached cells were collected and incubated with Alexa Fluor 647 annexin V Ready Flow Conjugate (Thermo Scientific) for $15 \mathrm{~min}$ and 10,000 cells were analysed using a BD Accuri C6 flow cytometer.

\section{Statistical analysis}

The data presented represents the mean \pm SEM of at least three independent biological experiments. Data from each biological replicate was assessed for variance within the respective group and only data with similar variance between groups was included in the statistical analysis. One-way ANOVA with Dunnett's post-hoc test, two-way ANOVA with Bonferroni post-hoc test, or Student's $t$-test were used where appropriate to compare data to the control group; $" p<0.05,{ }^{* *} p<0.01,{ }^{* * * *} p<0.001,{ }^{* * * * * *} p<$ 0.0001 .

\footnotetext{
Acknowledgements

This work was supported by funding from The Humane Research Trust, UK, and we are grateful to the laboratory of Javier García-Sancho for providing the SERCA2b constructs.

\section{Author details}

${ }^{1}$ Department of Eye and Vision Science, Institute of Ageing and Chronic Disease, University of Liverpool, Liverpool L7 8TX, UK. ${ }^{2}$ Systems Microscopy Centre, Faculty of Biology, Medicine and Health, University of Manchester, Manchester M13 9PT, UK. ${ }^{3}$ School of Biological Sciences, Faculty of Biology, Medicine and Health, University of Manchester, Manchester M13 9PT, UK. ${ }^{4}$ Department of Cellular and Molecular Physiology, Institute of Translational Medicine, University of Liverpool, Liverpool L69 3BX, UK
}

Conflict of interest

The authors declare that they have no conflict of interest. 


\section{Publisher's note}

Springer Nature remains neutral with regard to jurisdictional claims in published maps and institutional affiliations.

The online version of this article (https://doi.org/10.1038/s41420-019-0212-4) contains supplementary material, which is available to authorized users.

Received: 19 June 2019 Revised: 24 July 2019 Accepted: 29 July 2019 Published online: 03 September 2019

\section{References}

1. Attardi, L. D. et al. PERP, an apoptosis-associated target ofp53, is a novel member of the PMP-22/gas3 family. Genes Dev. 14, 704-718 (2000).

2. Ihrie, R. A. et al. Perp is a mediator of p53-dependent apoptosis in diverse cell types. Curr. Biol. 13, 1985-1990 (2003).

3. Ihrie, R. A. et al. Perp is a p63-regulated gene essential for epithelial integrity. Cell 120, 843-856 (2005).

4. Flores, E. R. et al. p63 and p73 are required for p53-dependent apoptosis in response to DNA damage. Nature 416, 560-564 (2002).

5. Davies, L., Spiller, D., White, M. R. H., Grierson, I. \& Paraoan, L. PERP expression stabilizes active p53 via modulation of p53-MDM2 interaction in uveal melanoma cells. Cell Death Dis. 2, e136 (2011).

6. Davies, L. et al. P53 apoptosis mediator PERP: localization, function and caspase activation in uveal melanoma. J. Cell. Mol. Med. 13, 1995-2007 (2009).

7. Ihrie, R. A. \& Attardi, L. D. Perp-etrating p53-dependent apoptosis. Cell Cycle 3, 265-267 (2004).

8. Berridge, M. J., Bootman, M. D. \& Roderick, H. L. Calcium signalling: dynamics, homeostasis and remodelling. Nat. Rev. Mol. Cell Biol. 4, 517-529 (2003).

9. Vandecaetsbeek, l., Vangheluwe, P., Raeymaekers, L., Wuytack, F. \& Vanoevelen, J. The $\mathrm{Ca}^{(2+)}$ pumps of the endoplasmic reticulum and golgi apparatus. Cold Spring Harb. Perspect. Biol. 3, a004184 (2011).

10. Brini, M. \& Carafoli, E. Calcium pumps in health and disease. Physiol. Rev. 89, 1341-1378 (2009).

11. Manjarres, I. M., Rodriguez-Garcia, A., Alonso, M. T. \& Garcia-Sancho, J. The sarco/endoplasmic reticulum $\mathrm{Ca}^{(2+)}$ ATPase (SERCA) is the third element in capacitative calcium entry. Cell Calcium 47, 412-418 (2010).

12. Szegezdi, E., Logue, S. E., Gorman, A. M. \& Samali, A. Mediators of endoplasmic reticulum stress-induced apoptosis. EMBO Rep. 7, 880-885 (2006).

13. Rutkowski, D. T. \& Kaufman, R. J. A trip to the ER: coping with stress. Trends Cell Biol. 14, 20-28 (2004).

14. Caspersen, C., Pedersen, P. S. \& Treiman, M. The sarco/endoplasmic reticulum calcium-ATPase $2 \mathrm{~b}$ is an endoplasmic reticulum stress-inducible protein. J. Biol. Chem. 275, 22363-22372 (2000).

15. Højmann Larsen, A., Frandsen, A. \& Treiman, M. Upregulation of the SERCAtype $\mathrm{Ca}(2+)$ pump activity in response to endoplasmic reticulum stress in PC12 cells. BMC Biochem. 2, 4 (2001).

16. Kuo, T. H. et al. Co-ordinated regulation of the plasma membrane calcium pump and the sarco(endo)plasmic reticular calcium pump gene expression by $\mathrm{Ca}^{2+}$. Cell Calcium 21, 399-408 (1997).

17. Yorimitsu, T., Nair, U., Yang, Z. \& Klionsky, D. J. Endoplasmic reticulum stress triggers autophagy. J. Biol. Chem. 281, 30299-30304 (2006).

18. Deegan, S., Saveljeva, S., Gorman, A. M. \& Samali, A. Stress-induced self-cannibalism: on the regulation of autophagy by endoplasmic reticulum stress. Cell. Mol. Life Sci. 70, 2425-2441 (2013).

19. Liu, Z., LV, Y., Zhao, N., Guan, G. \& Wang, J. Protein kinase R-like ER kinase and its role in endoplasmic reticulum stress-decided cell fate. Cell Death Dis. $\mathbf{6}$, e1822 (2015).

20. Rizzuto, R. et al. Close contacts with the endoplasmic reticulum as determinants of mitochondrial $\mathrm{Ca}^{2+}$ responses. Science 280, 1763-1766 (1998).
21. Pinton, P., Giorgi, C., Siviero, R., Zecchini, E. \& Rizzuto, R. Calcium and apoptosis: ER-mitochondria $\mathrm{Ca}^{2+}$ transfer in the control of apoptosis. Oncogene 27, 6407-6418 (2008).

22. Mendes, C. C. P. et al. The type III inositol 1,4,5-trisphosphate receptor preferentially transmits apoptotic $\mathrm{Ca}^{2+}$ signals into mitochondria. J. Biol. Chem. 280, 40892-40900 (2005).

23. Flourakis, M. et al. Orai1 contributes to the establishment of an apoptosisresistant phenotype in prostate cancer cells. Cell Death Dis. 1, e75 (2010).

24. Marchi, S. et al. Mitochondrial and endoplasmic reticulum calcium homeostasis and cell death. Cell Calcium 69, 62-72 (2017).

25. Ma, T. S., Mann, D. L., Lee, J. H. \& Gallinghouse, G. J. SR compartment calcium and cell apoptosis in SERCA overexpression. Cell Calcium 26, 25-36 (1999).

26. Chami, M. et al. Serca1 truncated proteins unable to pump calcium reduce the endoplasmic reticulum calcium concentration and induce apoptosis. J. Cell Biol. 153, 1301-1314 (2001).

27. Chami, M. et al. Role of SERCA1 truncated isoform in the proapoptotic calcium transfer from ER to mitochondria during ER stress. Mol. Cell 32, 641-651 (2008).

28. Sehgal, P. et al. Inhibition of the sarco/endoplasmic reticulum (ER) $\mathrm{Ca}^{2+}$-ATPase by thapsigargin analogs induces cell death via $\mathrm{ER} \mathrm{Ca}^{2+}$ depletion and the unfolded protein response. J. Biol. Chem. 292, 19656-19673 (2017).

29. Miller, S. G., Carnell, L. \& Moore, H. H. Post-Golgi membrane traffic: brefeldin A inhibits export from distal Golgi compartments to the cell surface but not recycling. J. Cell Biol. 118, 267-283 (1992).

30. Bunz, F. et al. Requirement for p53 and p21 to sustain G2 arrest after DNA damage. Science 282, 1497-1501 (1998).

31. Sui, X. et al. p53 suppresses stress-induced cellular senescence via regulation of autophagy under the deprivation of serum. Mol. Med. Rep. 11, 1214-1220 (2014).

32. Franke, W. et al. Transmembrane protein PERP is a component of tessellate junctions and of other junctional and non-junctional plasma membrane regions in diverse epithelial and epithelium-derived cells. Cell Tissue Res. 353, 99-115 (2013).

33. Birmingham, C. L., Smith, A. C., Bakowski, M. A., Yoshimori, T. \& Brumell, J. H. Autophagy controls salmonella infection in response to damage to the Salmonella-containing vacuole. J. Biol. Chem. 281, 11374-11383 (2006).

34. Wang, L., Yan, J., Niu, H., Huang, R. \& Wu, S. Autophagy and ubiquitination in Salmonella infection and the related inflammatory responses. Front. Cell. Infect. Micobiol. 8, 78 (2018).

35. Hallstrom, K. N. et al. PERP, a host tetraspanning membrane protein, is required for Salmonella-induced inflammation. Cell. Microbiol. 17, 843-859 (2015).

36. Booth, L. A., Tavallai, S., Hamed, H. A., Cruickshanks, N. \& Dent, P. The role of cell signalling in the crosstalk between autophagy and apoptosis. Cell. Signal. 26, 549-555 (2014).

37. Okeke, E., Dingsdale, H., Parker, T., Voronina, S. \& Tepikin, A. V. Endoplasmic reticulum-plasma membrane junctions: structure, function and dynamics. J. Physiol. 594, 2837-2847 (2016).

38. Chemaly, E. R., Troncone, L. \& Lebeche, D. SERCA control of cell death and survival. Cell Calcium 69, 46-61 (2018).

39. Giorgi, C. et al. p53 at the endoplasmic reticulum regulates apoptosis in a $\mathrm{Ca}^{2+}$-dependent manner. Proc. Natl Acad. Sci. USA 112, 1779-1784 (2015).

40. Hewarathna, A., Dremina, E. \& Schöneich, C. Inhibition and conformational change of SERCA3b induced by Bcl-2. Biochim. Biophys. Acta 1865, 121-131 (2017).

41. Pinton, P. et al. The $\mathrm{Ca}^{2+}$ concentration of the endoplasmic reticulum is a key determinant of ceramide-induced apoptosis: significance for the molecular mechanism of BCl-2 action. EMBO J. 20, 2690-2701 (2001).

42. Singaravelu, K., Devalaraja-Narashimha, K., Lastovica, B. \& Padanilam, B. J. PERP, a p53 proapoptotic target, mediates apoptotic cell death in renal ischemia. Am. J. Physiol. Renal Physiol. 296, F847-F858 (2009).

43. Awais, R., Spiller, D. G., White, M. R. H. \& Paraoan, L. p63 is required beside p53 for PERP-mediated apoptosis in uveal melanoma. Br. J. Cancer 115, 983-992 (2016). 